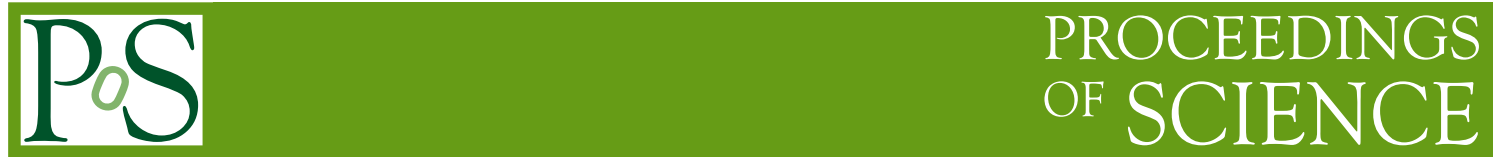

\title{
The Hagedorn thermostat
}

\section{G. Moretto*, L. Phair}

Nuclear Science Division, Lawrence Berkeley National Laboratory, Berkeley, CA, USA

E-mail: LGMoretto@lbl.gov, LWPhairelbl.gov

\section{K. A. Bugaev}

Bogolyubov Institute for Theoretical Physics, Kiev, Ukraine

E-mail: bugaeveth.physik.uni-frankfurt.de

\section{J. B. Elliott}

Lawrence Livermore National Laboratory

E-mail: elliott38@llnl.gov

\begin{abstract}
A system $\mathscr{H}$ with a Hagedorn-like mass spectrum can sustain the unique temperature $T_{\mathscr{H}}$ encoded in the spectrum itself. $\mathscr{H}$ imposes the same temperature to all emitted particles which are then in physical and chemical equilibrium with $\mathscr{H}$ and with each other. Coexistence and phase transition between hadronic and partonic phases are thus completely characterized. This may explain the recurring constant physical and chemical temperature observed in several experiments. The near indifference of $\mathscr{H}$ to fragmentation or coalescence makes this approach relevant to heavy ion and elementary particle collisions alike. The equation of state for a gas of $\mathscr{H}$ systems has been derived. The introduction of a bag surface energy makes the equilibrium bag temperature dependent on the radius, the smallest bags being hottest. A temperature dependence of the bag's surface energy leads naturally to a critical point.
\end{abstract}

The 3rd edition of the InternationalÂăWorkshop — The Critical Point and Onset of Deconfinement July 3-7 2006

Galileo Galilei Institute, Florence, Italy

\footnotetext{
* Speaker.
} 


\section{Introduction}

According to Hagedorn, the hadronic mass spectrum (level density) has the asymptotic $(m \rightarrow$ $\infty)$ form

$$
\rho_{\mathscr{H}}(m) \approx \exp \left(m / T_{\mathscr{H}}\right)
$$

where $m$ is the mass of the hadron in question and $T_{\mathscr{H}}$ is the parameter (temperature) controlling the exponential rise of the mass spectrum $[1,2]$. The question of the mass range over which (1.1) is valid is still under discussion [3].

The M.I.T. bag model [4] of partonic matter leads to the same spectrum via a constant pressure $B$ of the containing "bag" $[5,6]$. In the absence of conserved charges the bag pressure $B$ forces a constant temperature $T_{B}$ and energy density $\varepsilon$ from which it follows that the bag entropy is

$$
S=\varepsilon V / T_{B}=m / T_{B}
$$

where $V$ and $m$ are the volume and mass of the bag respectively. Thus the bag mass spectrum $\exp (S)$ is identical to Eq. $(1.1)[5,6]$. This property implies the lack of any surface energy associated with the bag.

A variety of experiments with high energy $(\sqrt{s} \geq 30-50 \mathrm{GeV})$ elementary particle collisions on very different systems indicate a constant temperature characterizing both chemical and physical equilibrium at vanishing baryonic densities $[7,8,9]$. It is interesting to explore the connection of these empirical temperatures with the Hagedorn temperature $T_{\mathscr{H}}$ on one hand and the bag temperature $T_{B}$ on the other [10].

We will show that the temperature of any such $\mathscr{H}$ system is not affected by the extrinsic injection of energy into the system but it is encoded and strictly enforced by the fixed temperature of the mass spectrum.

The insertion of an exponential spectrum such as Eq. (1.1) in the partition function

$$
\mathscr{Z}(T)=\int_{E_{\min }}^{\infty} \rho_{\mathscr{H}}(E) e^{-\frac{E}{T}} d E
$$

led to the incorrect conclusion that the entire range of temperatures $0 \leq T<T_{\mathscr{H}}$ is accessible and that $T_{\mathscr{H}}$ is the limiting temperature of the system.

In order to see the origin of this erroneous conclusion, let us consider the following illuminating exercise. Consider a system $A$ composed of ice and water at standard pressure (see Fig. 1). For such a system the temperature (kelvin) is $T_{A}=273 \mathrm{~K}$. Because of coexistence, we can feed or extract heat to/from the system without changing $T_{A}$. This is a strict thermodynamic requirement: we say that the system $A$ is a thermostat.

If a quantity $Q$ of heat is added to the sytem, the change in entropy is

$$
\Delta S=Q / T_{A}
$$

The level density of $A$ is then

$$
\rho(Q)=S_{0} e^{Q / T_{A}} \approx K e^{E / T_{A}} .
$$




\section{Can a "thermostat" have a temperature other than its own?}

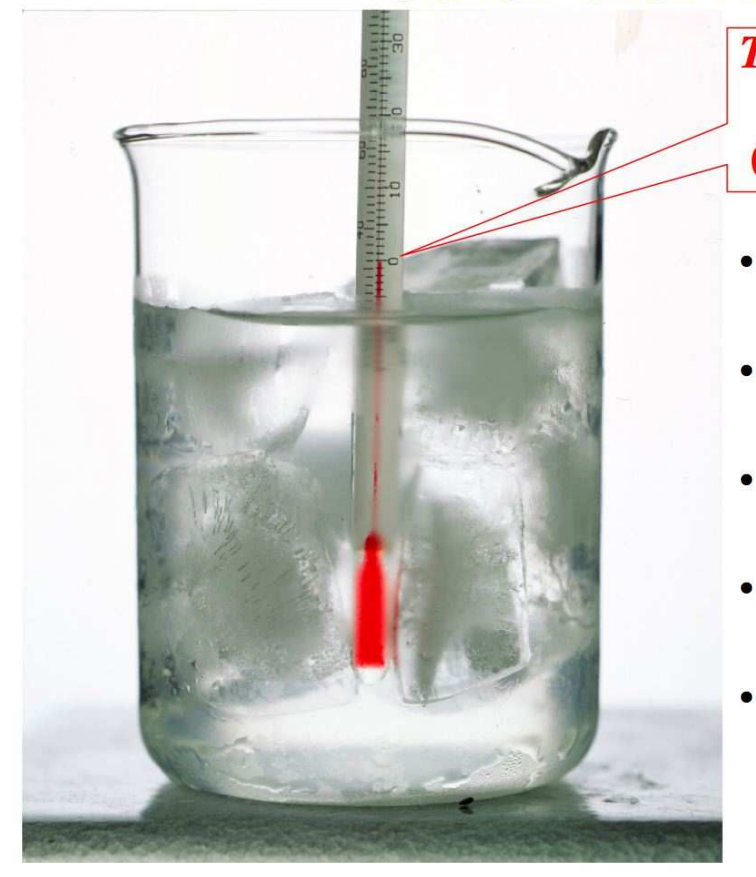

$T=T_{c}=273 \mathrm{~K}$

or

$0 \leq T \leq 273 K$

- $S=S_{0}+\frac{\Delta Q}{T}=S_{0}+\frac{E}{T_{0}}$

- $\rho(E)=e^{S}=e^{S_{0}+\frac{E}{T_{0}}}$

- Is $T_{0}$ just a "parameter"?

- $Z(T)=\int d E \rho(E) e^{-E / T}=\frac{T_{0} T}{T_{0}-T} e^{S_{0}}$

- According to this, a thermostat, can have any temperature lower than its own!

Figure 1: Example of ice and water at coexistence and the resulting (erroneous) partition function.

The level density, or spectrum, is exponential in $E$ and depends only on the intrinsic "parameter" $T_{A}$. Let us calculate the partition function of $A$ :

$$
Z(T)=\int e^{E / T_{A}} e^{-E / T} d E=\int e^{-\left(\frac{1}{T}-\frac{1}{T_{A}}\right) E} d E=\frac{T_{A} T}{T_{A}-T}
$$

This seems to indicate that $A$ can assume any temperature $0 \leq T<T_{A}$. This violates thermodynamics, which requires that the only temperature possible for $A$ is $T_{A}$. What is the trouble?

Let us consider two systems $A, B$ with level densities $\rho_{A}$ and $\rho_{B}$. Let the systems be thermally coupled to each other with total energy $E$. We now calculate the distribution in energies between the two systems,

$$
\rho_{T}(x)=\rho_{A}(E-x) \rho_{B}(x)
$$

Let $A$ be a "thermostat", i.e. $\rho_{A}=e^{\varepsilon / T_{A}}$. Then

$$
\rho_{T}(x)=\exp \left(\frac{E-x}{T_{A}}\right) \rho_{B}(x)=e^{E / T_{A}} e^{-x / T_{A}} \rho_{B}(x)
$$


Let us integrate over $x$

$$
\int \rho_{T}(x) d x=e^{E / T_{A}} \int e^{-x / T_{A}} \rho_{B}(x) d x=e^{E / T_{A}} Z_{B}\left(T_{A}\right) .
$$

This is the thermodynamic justification of the partition function $Z_{B}\left(T_{A}\right)$ and the meaning of "implicit" thermostat. By changing "thermostat" we can change $T_{A}$ and the temperature of $B$.

Thus, every time we construct a partition function, we imply the gedanken experiment of connecting the system to a thermostat, and that this experiment is actually possible for the system we are studying. Does this always work?

To see this, let us look for the most probable value of the distribution $\rho_{T}(x)$, which defines the equilibrium partition, by taking the log and differentiating:

$$
\begin{aligned}
\ln \rho_{T}(x) & =\ln \rho_{A}(E-x)+\ln \rho_{B}(x) \\
\partial \ln \rho_{T}(x) / \partial x & =-\partial \ln \rho_{A} / \partial x\left|+\partial \ln \rho_{B} / \partial x\right|=0 \quad \Leftrightarrow \quad 1 / T_{A}=1 / T_{B} .
\end{aligned}
$$

For this to be possible, it is necessary that $\rho_{A}$ and $\rho_{B}$ admit the same logarithmic derivative somewhere in the allowed range of energy $x$ (see Fig. 2).

Usually, and always for concave functions, $S(x)=\ln \rho(x)$ and $T=(\partial S / \partial x)^{-1}$ is such that $0 \leq T \leq \infty$. Thus, for such systems it is possible to match derivatives for whatever value of $E$. Thermal equilibrium is achievable over a broad range of temperatures.

However, if $S_{A}(E)=\ln \rho_{A}(E)$ is linear in $E$, then $T_{A}=(\partial S / \partial E)^{-1}$ is a constant, independent of $E$. In this case, it is up to $B$ to look for the value of $x$ at which its logarithmic derivative matches $1 / T_{A}$. The system $A$ is a "thermostat" at $T=T_{A}$ and $B$ can only try to assume the value $T=T_{B}=T_{A}$, if it can do so.

Now suppose that also $S_{B}(E)=\ln \rho_{B}(E)$ is linear in $E$ with an inverse slope $T_{B}$. This means that only if $T_{A}=T_{B}$ is equilibrium possible, and the partition function of $B, Z_{B}$ is meaningfully defined only for $T=T_{B}$ and not for $0 \leq T \leq T_{B}$. We cannot force a temperature $T \neq T_{B}$ on a thermostat. It can only have its own intrinsic temperature $T_{B}$. These arguments are summarized graphically in Fig. 2.

Placing systems $A$ and $B$ into contact will lead to a continuous heat flow from one system to the other. Thermal equilibrium is not achievable.

Summarizing: it is permissible to calculate a system's partition function only if its $S(E)$ admits as inverse derivatives values such as we want to impose through our Laplace transform. Failing that, the resulting partition function does not satisfy any thermodynamic criterion.

Carlitz noticed [11] that Eq. (1.1) leads to a nonequivalence between the (grand)canonical and microcanonical descriptions. However, the striking consequences of this fact were not appreciated.

We show here that the exponential form of the mass spectrum in Eq. (1.1) forces the unique temperature $T_{\mathscr{H}}$ on both the chemical and physical equilibria associated with it. Below we explore the consequences of this hitherto unappreciated fact.

To begin, we show that a system $\mathscr{H}$ possessing a Hagedorn-like spectrum, characterized by an entropy of the form (1.2), not only has a unique microcanonical temperature $T_{\mathscr{H}}$

$$
T_{\mathscr{H}}=(d S / d E)^{-1}=T_{B},
$$




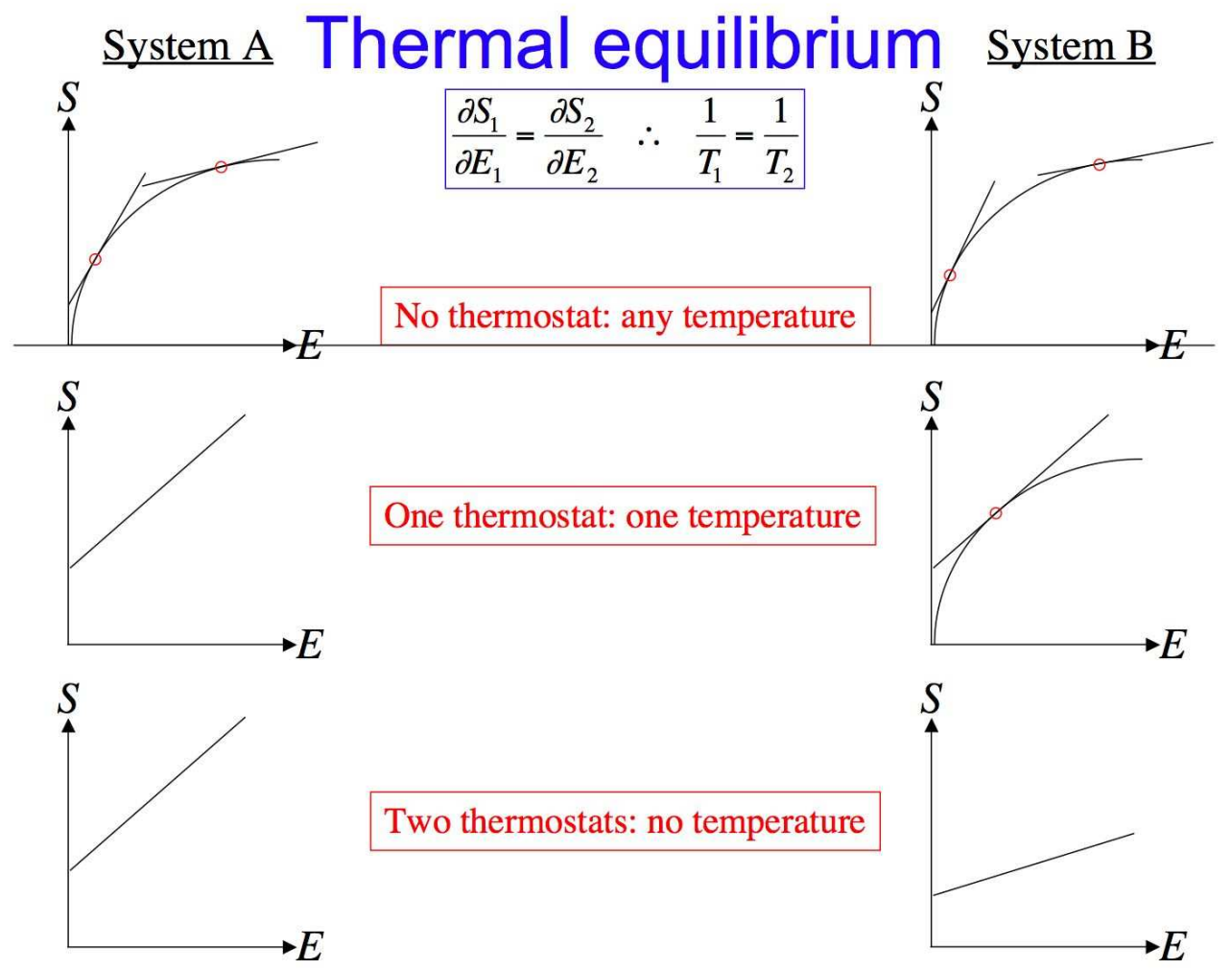

Figure 2: Consequences of placing systems A and B in thermal contact when neither (top), one (middle), or both (bottom) are thermostats.

but also imparts this same temperature to any other system to which $\mathscr{H}$ is coupled. In the language of thermodynamics: $\mathscr{H}$ is a perfect thermostat with the constant temperature $T_{\mathscr{H}}$.

Incidentally, it is worth noting that a perfect thermostat is indifferent to the transfer of any portion of its energy to any parcel within itself, no matter how small. In other words, it is at the limit of phase stability and the internal fluctuations of its energy density are maximal. Therefore it does not matter whether this thermostat is one large bag or it is fragmented in an arbitrary number of smaller bags or, equivalently, it is a system of hadrons with a spectrum given by Eq. (1.1). This has consequences on the properties of $\mathscr{H}$ as we shall see below.

\section{Harmonic Oscillator Coupled to $\mathscr{H}$}

In order to demonstrate the thermostatic behavior of a Hagedorn system, let us begin by coupling $\mathscr{H}$ to a one dimensional harmonic oscillator and use a microcanonical treatment. The unnormalized probability $P(\varepsilon)$ for finding an excitation energy $\varepsilon$ in the harmonic oscillator out of the 
system's total energy $E$ is

$$
P(\varepsilon) \sim \rho_{\mathscr{H}}(E-\varepsilon) \rho_{\text {osc }}(\varepsilon)=\exp \left(\frac{E-\varepsilon}{T_{\mathscr{H}}}\right)=\rho_{\mathscr{H}}(E) \exp \left(-\frac{\varepsilon}{T_{\mathscr{H}}}\right) .
$$

Recall that for a one dimensional harmonic oscillator $\rho_{\text {osc }}$ is a constant. As the above equation shows, the energy spectrum of the oscillator is canonical (Boltzmann factor!) up to the upper limit $\varepsilon_{\max }=E$ with an inverse slope (temperature) of $T_{\mathscr{H}}$ independent of $E$. The mean value of the energy of the oscillator is

$$
\bar{\varepsilon}=T_{\mathscr{H}}\left[1-\frac{E / T_{\mathscr{H}}}{\exp \left(E / T_{\mathscr{H}}\right)-1}\right] .
$$

Thus in the limit that $E \rightarrow \infty: \bar{\varepsilon} \rightarrow T_{\mathscr{H}}$, no temperature other than $T_{\mathscr{H}}$ is admitted. This example shows that a one dimensional harmonic oscillator can be used as an ideal thermometer.

\section{An ideal vapor coupled to $\mathscr{H}$}

For a physically more relevant example, let us consider a vapor of $N \gg 1$ non-interacting particles of mass $m$ coupled to $\mathscr{H}$. The microcanonical level density of the vapor with kinetic energy $\varepsilon$ is

$$
\rho_{\text {vapor }}(\varepsilon)=\frac{V^{N}}{N !\left(\frac{3}{2} N\right) !}\left(\frac{m \varepsilon}{2 \pi}\right)^{\frac{3}{2} N},
$$

where $V$ is the volume. The microcanonical partition of the total system is

$$
\rho_{\text {total }}(E, \varepsilon)=\rho_{\mathscr{H}}(E-\varepsilon) \rho_{\text {vapor }}(\varepsilon)=\frac{V^{N}}{N !\left(\frac{3}{2} N\right) !}\left(\frac{m \varepsilon}{2 \pi}\right)^{\frac{3}{2} N} e^{\frac{E-m N-\varepsilon}{T_{\mathscr{H}}}} .
$$

Again, the distribution of the vapor is exactly canonical up to $\varepsilon_{\max }=E$, if the particles are independently present, or $\varepsilon_{\max }=E-m N$, if the particles are generated by $\mathscr{H}$. In either case, the temperature of the vapor is always $T_{\mathscr{H}}$.

At fixed $N$ the maximum of $\rho_{\text {total }}(E, \varepsilon)$ with respect to $\varepsilon$ gives the most probable kinetic energy per particle as

$$
\frac{\partial \ln \rho_{\text {total }}(E, \varepsilon)}{\partial \varepsilon}=\frac{3 N}{2 \varepsilon}-\frac{1}{T_{\mathscr{H}}}=0 \Rightarrow \frac{\varepsilon}{N}=\frac{3}{2} T_{\mathscr{H}},
$$

provided that $E \geq m N+\frac{3}{2} N T_{\mathscr{H}}$. For $m N<E<m N+\frac{3}{2} N T_{\mathscr{H}}$, the most probable kinetic energy per particle value is $\frac{\varepsilon}{N}=\frac{E}{N}-m<\frac{3}{2} T_{\mathscr{H}}$; for $E \leq m N, \frac{\varepsilon}{N}=0 . T_{\mathscr{H}}$ is the sole temperature characterizing the distribution up to the microcanonical cut off, which may be above or below the maximum of the distribution, since the form of $\rho_{\text {total }}(E, \varepsilon)$ is $E$-independent.

The maximum of $\rho_{\text {total }}(E, \varepsilon)$ with respect to $N$ at fixed $V$ is given by

$$
\frac{\partial \ln \rho_{\text {total }}(E, \varepsilon)}{\partial N}=-\frac{m}{T_{\mathscr{H}}}+\ln \left[\frac{V}{N}\left(\frac{m T_{\mathscr{H}}}{2 \pi}\right)^{\frac{3}{2}}\right]=0,
$$

where Eq. (3.3) was used for $\varepsilon$. Thus the most probable particle density of the vapor is independent of $V$ :

$$
\frac{N}{V}=\left(\frac{m T_{\mathscr{H}}}{2 \pi}\right)^{\frac{3}{2}} e^{-\frac{m}{T_{\mathscr{H}}}} \equiv n_{\mathscr{H}}
$$


This most important result shows that not only is $\mathscr{H}$ a perfect thermostat, but also a perfect particle reservoir. Different particles of different mass $m$ will be automatically in chemical equilibrium with each other. At equilibrium, particles emitted from $\mathscr{H}$ form a saturated vapor at coexistence with $\mathscr{H}$ at temperature $T_{\mathscr{H}}$. This describes a first order phase transition (hadronic to partonic). Coexistence occurs at a single temperature fixed by the bag pressure. Different arguments lead to a similar conclusion concerning the existence of a phase transition [11].

These key results may explain the common value of: the hadronization temperatures obtained within the statistical hadronization model at vanishing baryonic densities [9]; the inverse slopes of the transverse mass spectra of hadrons observed in high energy elementary particle collisions with the transverse momenta $p_{T} \leq 1 \mathrm{GeV}[7,8]$; and the transition temperature from lattice QCD calculations for low baryonic density [12]. For further discussion see [13].

Let us consider the case in which the vapor particle mass $m$ is part of a distribution of masses. We want to determine the most probable mass respresented in the vapor. The system's level density $\rho_{\text {total }}(E, \varepsilon)$ is still given by Eq. (3.2). Using Eqs. (3.3) and (3.5), one finds the most probable value of the system's level density as $\rho_{\text {total }}^{*}(E, \varepsilon) \approx \exp \left[S^{*}\right]$, where the entropy is $S^{*}=E / T_{\mathscr{H}}+N$. Differentiating $\rho_{\text {total }}^{*}(E, \varepsilon)$ with respect to $m$ and applying Eq. (3.5) gives

$$
\frac{\partial \ln \rho_{\text {total }}^{*}(E, \varepsilon)}{\partial m}=N\left[\frac{3}{2 m}-\frac{1}{T_{\mathscr{H}}}\right]=0 \Rightarrow m=\frac{3}{2} T_{\mathscr{H}},
$$

i.e. the last equality provides the maximum of level density for $N \neq 0$.

Substituting the most probable value of $\varepsilon$ and $m$ into the most probable value of $N$ gives the vapor concentration

$$
\frac{N}{V}=\left(\frac{3}{4 \pi e}\right)^{\frac{3}{2}} T_{\mathscr{H}}^{3}
$$

If the mass given by Eq. (3.6) does not exist among the available masses, then the level density's most probable value $\rho_{\text {total }}^{*}(E, \varepsilon)$ corresponds to the mass $m^{*}$ nearest to $\frac{3}{2} T_{\mathscr{H}}$ and $N\left(m^{*}\right)$ given by Eq. (3.5). The value of $m^{*}$ that maximizes the level density $\rho_{\text {total }}^{*}(E, \varepsilon)$ is the pion mass.

\section{Hagedorn Thermostat Model}

Let us consider the microcanonical ensemble of $N_{B}$ Boltzmann point-like particles of mass $m_{B}$ and degeneracy $g_{B}$, and $N_{H}$ hadronic point-like resonances of mass $m_{H}$ with a mass spectrum $g_{H}\left(m_{H}\right)=\exp \left[m_{H} / T_{H}\right]\left(m_{\mathrm{o}} / m_{H}\right)^{a}$ for $m_{H} \geq m_{\mathrm{o}}$ which obeys the inequalities $m_{\mathrm{o}} \gg T_{H}$ and $m_{\mathrm{o}}>m_{B}$. A recent analysis [14] suggests that the Hagedorn mass spectrum can be established for $m_{\mathrm{o}}<2$ $\mathrm{GeV}$.

In the SBM [15] and the MIT bag model [5] it was found that for $m_{H} \rightarrow \infty$ the parameter $a \leq 3$. For finite resonance masses the value of $a$ is unknown, so it will be considered as a fixed parameter.

The microcanonical partition of the system, with volume $V$, total energy $U$ and zero total momentum, can be written as follows

$$
\Omega=\frac{V^{N_{H}}}{N_{H} !}\left[\prod_{k=1}^{N_{H}} g_{H}\left(m_{H}\right) \int \frac{d^{3} Q_{k}}{(2 \pi)^{3}}\right] \frac{V^{N_{B}}}{N_{B} !}\left[\prod_{l=1}^{N_{B}} g_{B} \int \frac{d^{3} p_{l}}{(2 \pi)^{3}}\right]
$$




$$
\delta\left(U-\sum_{i=1}^{N_{H}} \varepsilon_{i}^{H}-\sum_{j=1}^{N_{B}} \varepsilon_{j}^{B}\right),
$$

where the quantity $\varepsilon_{i}^{H}=\varepsilon\left(m_{H}, Q_{i}\right)\left(\varepsilon_{j}^{B}=\varepsilon\left(m_{B}, p_{j}\right)\right.$ and $\left.\varepsilon(M, P) \equiv \sqrt{M^{2}+P^{2}}\right)$ denotes the energy of the Hagedorn (Boltzmann) particle with the 3-momentum $\vec{Q}_{i}\left(\vec{p}_{j}\right)$. In order to simplify the presentation of our idea, Eq. (4.1) accounts for energy conservation only and neglects momentum conservation.

The microcanonical partition (4.1) can be evaluated by the Laplace transform in total energy $U$ [16]. Then the momentum integrals in (4.1) are factorized and can be performed analytically. The inverse Laplace transform in the conjugate variable $\lambda$ can be done analytically for the nonrelativistic and ultrarelativistic approximations of the one-particle momentum distribution function $\left(K_{2}(z)\right.$ is the modified Bessel function)

$$
\begin{aligned}
\int_{0}^{\infty} \frac{d^{3} p e^{-\lambda \varepsilon(M, p)}}{(2 \pi)^{3}} & =\frac{M^{2}}{2 \pi^{2} \lambda} K_{2}(M \lambda) \\
& \approx \begin{cases}\frac{2}{\lambda^{3}} I_{2} e^{-M \lambda}, & M R e(\lambda) \gg 1,\end{cases}
\end{aligned}
$$

where the auxiliary integral can be expressed in terms of the gamma function as follows

$$
I_{b} \equiv \int_{0}^{\infty} \frac{d \xi}{(2 \pi)^{2}} \xi^{b} e^{-\xi}=\frac{\Gamma(b+1)}{(2 \pi)^{2}} .
$$

The actual Hagedorn spectrum conatins a pre-exponential factor and becomes established above a lower threshold $m_{0}$. We are considering here the implications of these two facts in a more formal way.

Since the formal steps of further evaluation are similar for both cases, we discuss in detail the nonrelativistic limit only, and later present the results for the other case. The nonrelativistic approximation $(\operatorname{MRe}(\lambda) \gg 1)$ for Eq. (4.1) is as follows

$$
\begin{aligned}
\Omega_{n r}= & \frac{\left[V g_{H}\left(m_{H}\right)\left[2 m_{H}\right]^{\frac{3}{2}} I_{\frac{1}{2}}\right]^{N_{H}}}{N_{H} !} \\
& \frac{\left[V g_{B}\left[2 m_{B}\right]^{\frac{3}{2}} I_{\frac{1}{2}}\right]^{N_{B}}}{N_{B} !} \frac{E_{k i n}^{\frac{3}{2}\left(N_{H}+N_{B}\right)-1}}{\left(\frac{3}{2}\left(N_{H}+N_{B}\right)-1\right) !},
\end{aligned}
$$

where $E_{k i n}=U-m_{H} N_{H}-m_{B} N_{B}$ is the kinetic energy of the system.

As shown below, the most realistic case corresponds to the nonrelativistic treatment of the Hagedorn resonances because the resulting temperature is much smaller than their masses. Therefore, it is sufficient to consider the ultrarelativistic limit for the Boltzmann particles only. In this case $(\operatorname{MRe}(\lambda) \ll 1)$ the equation $(4.1)$ can be approximated as

$$
\begin{aligned}
\Omega_{u r}= & \frac{\left[V g_{H}\left(m_{H}\right)\left[2 m_{H}\right]^{\frac{3}{2}} I_{\frac{1}{2}}\right]^{N_{H}}}{N_{H} !} \\
& \frac{\left[V g_{B} 2 I_{2}\right]^{N_{B}}}{N_{B} !} \frac{E_{k i n}^{\frac{3}{2}\left(N_{H}+2 N_{B}\right)-1}}{\left(\frac{3}{2}\left(N_{H}+2 N_{B}\right)-1\right) !},
\end{aligned}
$$


where the kinetic energy does not include the rest energy of the Boltzmann particles, i.e. $E_{k i n}=$ $U-m_{H} N_{H}$.

Within our assumptions the above results are general and can be used for any number of particles, provided $N_{H}+N_{B} \geq 2$. It is instructive to consider first the simplest case $N_{H}=1$. This oversimplified model, in which a Hagedorn thermostat is always present, allows us to study the problem rigorously. For $N_{H}=1$ and $N_{B} \gg 1$ we treat the mass of Hagedorn thermostat $m_{H}$ as a free parameter and determine the value which maximizes the entropy of the system. The solution $m_{H}^{*}>0$ of

$$
\begin{aligned}
& \frac{\delta \ln \Omega_{n r}\left(N_{H}=1\right)}{\delta m_{H}}= \\
& \frac{1}{T_{H}}+\left(\frac{3}{2}-a\right) \frac{1}{m_{H}^{*}}-\frac{3\left(N_{B}+1\right)}{2 E_{k i n}}=0
\end{aligned}
$$

provides the maximum of the system's entropy, if for $m_{H}=m_{H}^{*}$ the second derivative is negative

$$
\begin{aligned}
& \frac{\delta^{2} \ln \Omega_{n r}\left(N_{H}=1\right)}{\delta m_{H}^{2}}= \\
& -\left(\frac{3}{2}-a\right) \frac{1}{m_{H}^{* 2}}-\frac{3\left(N_{B}+1\right)}{2 E_{k i n}^{2}}<0 .
\end{aligned}
$$

If the inequality (4.7) is satisfied, then the extremum condition (4.6) defines the temperature of the system of $\left(N_{B}+1\right)$ nonrelativistic particles

$$
T^{*}\left(m_{H}^{*}\right) \equiv \frac{2 E_{k i n}}{3\left(N_{B}+1\right)}=\frac{T_{H}}{1+\left(\frac{3}{2}-a\right) \frac{T_{H}}{m_{H}^{*}}} .
$$

Thus, as $m_{H}^{*} \rightarrow \infty$ it follows that $T^{*}\left(m_{H}^{*}\right) \rightarrow T_{H}$, while for finite $m_{H}^{*} \gg T_{H}$ and $a>\frac{3}{2}\left(a<\frac{3}{2}\right)$ the temperature of the system is slightly larger (smaller) than the Hagedorn temperature, i.e. $T^{*}>T_{H}$ $\left(T^{*}<T_{H}\right)$. Formally, the temperature of the system in equation (4.8) may differ essentially from $T_{H}$ for a light thermostat, i.e. for $m_{H}^{*} \leq T_{H}$. However, it is assumed that the Hagedorn mass spectrum exists above the cut-off mass $m_{\mathrm{o}} \gg T_{H}$, thus $m^{*} \gg T_{H}$.

\section{The Role of the Mass Cut-off}

Now we study the effect of the mass cut-off of the Hagedorn spectrum on the inequality (4.7) in more detail. For $a \leq \frac{3}{2}$ the condition (4.7) is satisfied. For $a>\frac{3}{2}$ the inequality (4.7) is equivalent to

$$
\frac{m_{H}^{* 2}}{\left(a-\frac{3}{2}\right) T^{*}\left(m_{H}^{*}\right)}>\frac{3}{2}\left(N_{B}+1\right) T^{*}\left(m_{H}^{*}\right),
$$

which means that a Hagedorn thermostat should be massive compared to the kinetic energy of the system.

A more careful analysis shows that for a negative value of the determinant $D_{n r}\left(\tilde{N} \equiv N_{B}-\frac{2}{3} a\right)$

$$
\begin{aligned}
D_{n r} \equiv & \left(U-m_{B} N_{B}-\frac{3}{2} T_{H} \tilde{N}\right)^{2}- \\
& 4\left(a-\frac{3}{2}\right) T_{H}\left(U-m_{B} N_{B}\right)<0,
\end{aligned}
$$


equation (4.6) has two complex solutions, while for $D_{n r}=0$ there exists a single real solution of (4.6). Solving (5.2) for $\left(U-m_{B} N_{B}\right)$, shows that for $\tilde{N}>\frac{2}{3} a-1$, i.e. for $N_{B}>\frac{4}{3}, a-1$ the inequality (5.2) does not hold and $D_{n r}>0$. Therefore, in what follows we will assume that $N_{B}>\frac{4}{3} a-1$ and only analyze the case $D_{n r}>0$. For this case equation (4.6) has two real solutions

$$
m_{H}^{ \pm}=\frac{1}{2}\left[U-m_{B} N_{B}-\frac{3}{2} T_{H} \tilde{N} \pm \sqrt{D_{n r}}\right] .
$$

For $a \leq \frac{3}{2}$ only $m_{H}^{+}$solution is positive and corresponds to a maximum of the microcanonical partition $\Omega_{n r}$.

For $a>\frac{3}{2}$ both solutions of (4.6) are positive, but only $m_{H}^{+}$is a maximum. From the two limiting cases:

$$
\begin{aligned}
& \frac{\delta \ln \Omega_{n r}\left(N_{H}=1\right)}{\delta m_{H}} \approx\left(\frac{3}{2}-a\right) \frac{1}{m_{H}} \text { for } m_{H} \approx 0, \\
& \frac{\delta \ln \Omega_{n r}\left(N_{H}=1\right)}{\delta m_{H}} \approx \frac{3\left(N_{B}+1\right)}{2 E_{k i n}} \quad \text { for } \quad E_{k i n} \approx 0,
\end{aligned}
$$

and the fact that $m_{H}^{ \pm}$obey the inequalities

$$
0<m_{H}^{-} \leq m_{H}^{+}<U-m_{B} N_{B}
$$

it is clear that $m_{H}^{*}=m_{H}^{-}$is a local minimum of the microcanonical partition $\Omega_{n r}$, while $m_{H}^{*}=m_{H}^{+}$ is a local maximum of the partition $\Omega_{n r}$.

Using Eq. (5.3) for $m_{H}^{+}$, it is clear that for any value of $a$ the constraint $m_{H}^{+} \geq m_{\mathrm{o}}$ is equivalent to the inequality

$$
N_{B} \leq N_{B}^{k i n} \equiv \frac{U-\left[\frac{m_{\mathrm{o}}}{T_{H}}-a\right] T^{*}\left(m_{\mathrm{o}}\right)}{m_{B}+\frac{3}{2} T^{*}\left(m_{\mathrm{o}}\right)} .
$$

Thus, at fixed energy $U$ for all $N_{B} \leq N_{B}^{k i n}$ at $m_{H}^{*}=m_{H}^{+}$there is a local maximum of the microcanonical partition $\Omega_{n r}$ with the temperature $T=T^{*}\left(m_{H}^{+}\right)$. For $N_{B}>N_{B}^{k i n}$ the maximum of the partition $\Omega_{n r}$ cannot be reached due to the cut-off constraint and, consequently, the most probable state corresponds to $m_{H}=m_{\mathrm{o}}$ with $T \leq T^{*}\left(m_{\mathrm{o}}\right)$ from Eq. (4.8). In other words, for $N_{B}>N_{B}^{k i n}$ the amount of energy $U$ is insufficient for the mass of the Hagedorn thermostat to be above the cut-off $m_{\mathrm{o}}$ and simultaneously maintain the temperature of the Boltzmann particles according to Eq. (4.8). By assumption there is a single Hagedorn thermostat in the system, therefore, as $N_{B}$ grows the temperature of the system decreases from $T^{*}\left(m_{\mathrm{o}}\right)$ value. Thus, the equality (5.7) defines the kinematical limit for reaching the maximum of the microcanonical partition.

To prove that the maximum of the microcanonical partition at $m_{H}=m_{H}^{+}$is global it is sufficient to show that the constraint $m_{H}^{+} \geq m_{\mathrm{o}}$ is not consistent with the condition $m_{H}^{-}>m_{\mathrm{o}}$. For $a \leq \frac{3}{2}$ the maximum is global because for $0<m_{H}<m_{H}^{+}\left(m_{H}>m_{H}^{+}\right)$the partition $\Omega_{n r}\left(N_{H}=1, m_{H}\right)$ monotonically increases (decreases) with $m_{H}$. For $a>\frac{3}{2}$ it is clear that the maximum at $m_{H}=m_{H}^{+}$ is local, if the state with mass $m_{H}=m_{\mathrm{o}}$ is more probable, i.e. $\Omega_{n r}\left(N_{H}=1, m_{\mathrm{o}}\right)>\Omega_{n r}\left(N_{H}=1, m_{H}^{+}\right)$. Due to (5.6) this can occur, if $m_{H}^{-}>m_{\mathrm{o}}$. Substituting Eq. (5.3) into the last inequality, shows that this inequality reduces to the condition $N_{B}>N_{B}^{k i n}$. This contradicts the constraint $m_{H}^{+} \geq m_{\mathrm{o}}$ in the form of Eq. (5.7). Thus, the maximum of the microcanonical partition is global. 


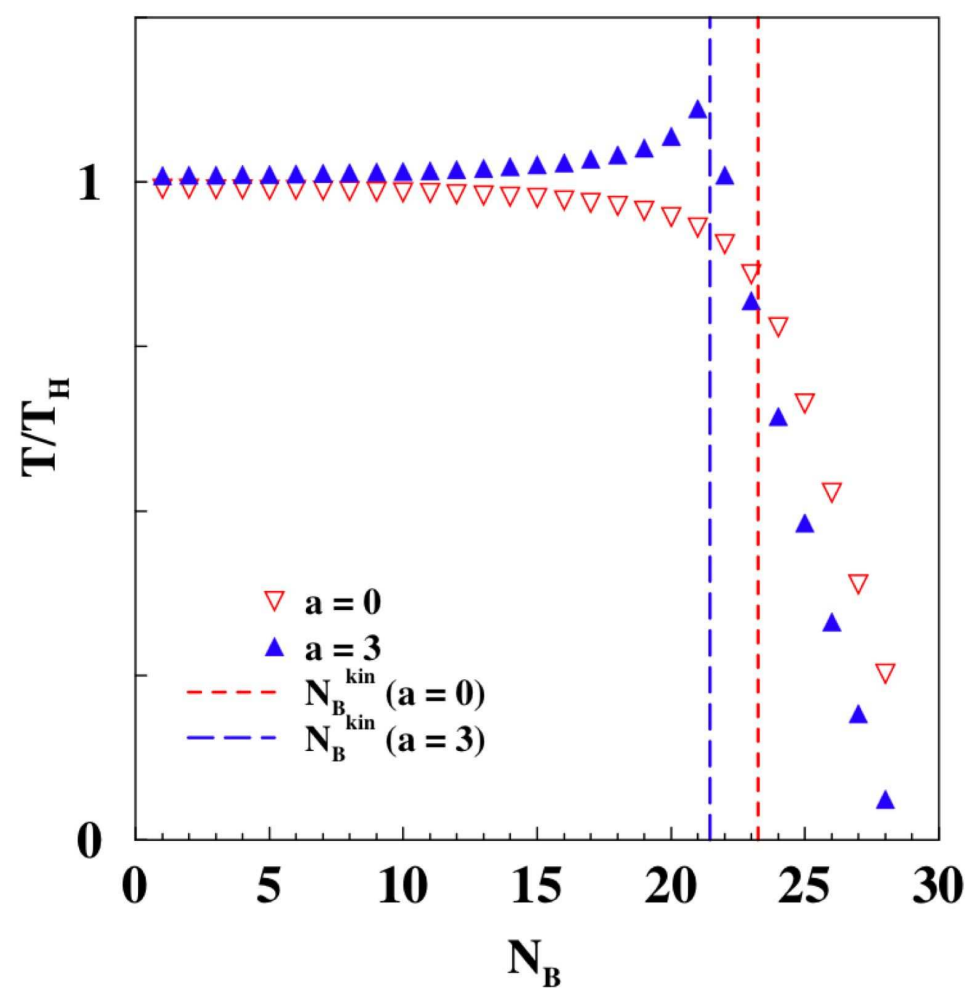

Figure 3: A typical behavior of the system's temperature as a function of the number of Boltzmann particles $N_{B}$ for $a=3$ and $a=0$ for the same value of the total energy $U=30 m_{B}$. Due to the thermostatic properties of a Hagedorn resonance, the system's temperature is nearly constant up to the kinematically allowed value $N_{B}^{k i n}$ given by Eq. (5.7).

To complete our consideration of the nonrelativistic case let us express the partition (4.4) in terms of the temperature (4.8). Applying the Stirling approximation to the factorial $\left(\frac{3}{2}\left(N_{B}+1\right)-1\right)$ ! for $N_{B}^{k i n}>N_{B} \gg 1$ and reversing the integral representations (4.2) and (4.3) for $\lambda=1 / T^{*}\left(m_{H}^{+}\right)$, one finds

$$
\begin{aligned}
& \Omega_{n r}\left(N_{H}=1\right)=\frac{V g_{H}\left(m_{H}^{+}\right)}{T^{*}\left(m_{H}^{+}\right)} \int \frac{d^{3} Q}{(2 \pi)^{3}} e^{-\frac{\sqrt{m_{H}^{+}+Q^{2}}}{T^{*}\left(m_{H}^{+}\right)}} \\
& \frac{e^{\frac{U}{T^{*}\left(m_{H}^{+}\right)}}}{N_{B} !}\left[V g_{B} \int \frac{d^{3} p}{(2 \pi)^{3}} e^{-\frac{\sqrt{m_{B}^{2}+p^{2}}}{T^{*}\left(m_{H}^{+}\right)}}\right]^{N_{B}} .
\end{aligned}
$$

This is just the grand canonical partition of $\left(N_{B}+1\right)$ Boltzmann particles with temperature $T^{*}\left(m_{H}^{+}\right)$. If $N_{B}>N_{B}^{k i n} \gg 1$, then $T^{*}\left(m_{H}^{+}\right)$in (5.8) should be replaced by

$$
T_{\mathrm{o}}\left(N_{B}\right) \equiv \frac{2\left(U-m_{B} N_{B}-m_{\mathrm{o}}\right)}{3\left(N_{B}+1\right)} .
$$


Fig. 3 shows that for $a>\frac{3}{2}$ the system's temperature $T=T^{*}\left(m_{H}^{+}\right)$as a function of $N_{B}$ remains almost constant for $N_{B}<N_{B}^{k i n}$, reaches a maximum at $N_{B}^{k i n}$ and rapidly decreases like $T=T_{\mathrm{o}}\left(N_{B}\right)$ for $N_{B}>N_{B}^{k i n}$. For $a<\frac{3}{2}$ the temperature has a plateau $T=T^{*}\left(m_{H}^{+}\right)$for $N_{B}<N_{B}^{k i n}$, and rapidly decreases for $N_{B}>N_{B}^{k i n}$ according to $T_{\mathrm{o}}\left(N_{B}\right)$.

The same results are valid for the ultrarelativistic treatment of Boltzmann particles. Comparing the nonrelativistic and ultrarelativistics expressions for the microcanonical partition, i.e. equations (4.4) and (4.5), respectively, one finds that the derivation of the ultrarelativistic limit requires only the substitution $N_{B} \rightarrow 2 N_{B}$ and $m_{B} / T_{H} \rightarrow 0$ in equations (4.6-5.8). Note that this substitution does not alter the expression for the temperature of the system, i.e. the right hand side of (4.8).

Finally, we show that for a heavy Hagedorn thermostat $\left(m_{H}^{+} \gg m_{\mathrm{o}}\right)$ these results remain valid for a single Hagedorn thermostat split into $N_{H}$ pieces of the same mass. Substituting $m_{H} \rightarrow m_{H} N_{H}$ in the nonrelativistic expressions (4.4) and minimizing it with respect to $m_{H}$, the temperature of the system in the form of equation (4.8) is $T^{*}\left(m_{H}^{*} N_{H}\right)$, where the mass of $N_{H}$ Hagedorn thermostats $m_{H}^{*}$ is related to the solution $m_{H}^{+}$of equation (5.3) as $m_{H}^{*}=m_{H}^{+} / N_{H}$. Since the original single thermostat of mass $m_{H}^{+}$was assumed to be heavy, it follows $T^{*}\left(m_{H}^{*} N_{H}\right)=T^{*}\left(m_{H}^{+}\right) \rightarrow T_{H}$. A more careful study using an exact expression for the microcanonical partition of $N_{H}$ Hagedorn thermostats of the same mass $m_{H}$ gives the same result, if $m_{H} \gg m_{\mathrm{o}}$. A generalization of these statements to the case of $N_{H}$ heavy Hagedorn thermostats of different masses also leads to the same result. Thus, splitting a single heavy Hagedorn thermostat into an arbitrary number of heavy resonances (heavier than $m_{\mathrm{o}}$ ) does not change the temperature of the system.

\section{6. $\mathscr{H}$ as a radiant bag}

Do the emitted particles need to remain in the proximity of $\mathscr{H}$ to insure equilibrium? Let us assume that $\mathscr{H}$ is a bag thick enough to absorb any given particle of the vapor striking it. Then, detailed balance requires that on average $\mathscr{H}$ radiates back the same particle. Under these conditions particles can be considered to be effectively emitted from the surface of $\mathscr{H}$. Thus the relevant fluxes do not depend in any way upon the inner structure of $\mathscr{H}$, nor on the presence of the outer vapor.

The results in equations (3.3) and (3.5) show that the saturated vapor concentration depends only on $m$ and $T_{\mathscr{H}}$ as long as $\mathscr{H}$ is present. A decrease in $V$ does not increase the vapor concentration, but induces a condensation of the corresponding amount of energy out of the vapor and into $\mathscr{H}$. An increase in $V$ keeps the vapor concentration constant via evaporation of the corresponding amount of energy out of $\mathscr{H}$ and into the vapor. This is reminiscent of liquid-vapor equilibrium at fixed temperature, except that here coexistence occurs at a single temperature $T_{\mathscr{H}}$, rather than over a range of temperatures as in ordinary fluids.

The bag wall is Janus faced: one side faces the partonic world, and, aside from conserved charges, radiates a partonic black body radiation responsible for balancing the bag pressure; the other side faces the hadronic world and radiates a hadronic black body radiation, mostly pions. Both sides of the bag wall are at temperature $T_{\mathscr{H}}$. It is tempting to attribute most, if not all, of the hadronic and partonic properties to the wall itself, possibly even the capability to enforce conservation laws globally (quantum number conductivity). Despite the fact that this wall is an 
insurmountable horizon, hadronic measurements such as bag size and total radiance can yield some properties of the partonic world, e.g. the number of degrees of freedom [7].

We can estimate an upper limit for the emission time using the outward energy flux of particles radiated from the bag. At equilibrium the in-going and out-going fluxes must be the same. The outward flux of particles $n_{\mathscr{H}}$ together with the energy flux $\varphi_{E_{\mathscr{H}}}$ and momentum flux $p_{\text {rad }}$ can be found using the techniques of $[17,18]$ and in the nonrelativistic approximation using Eq. (3.5) can be cast as

$$
\varphi_{n_{\mathscr{H}}} \simeq \frac{n_{\mathscr{H}}}{4}\left(\frac{m}{m+2 T_{\mathscr{H}}}\right) \sqrt{8 \frac{T_{\mathscr{H}}}{\pi m}}, \quad \varphi_{E_{\mathscr{H}}} \simeq\left(m+2 T_{\mathscr{H}}\right) \varphi_{n_{\mathscr{H}}}, \quad p_{\mathrm{rad}}=n_{\mathscr{H}} T_{\mathscr{H}} / 2
$$

The pressure $p_{\text {rad }}$ exerted on the bag by its radiation can be compared to the intrinsic bag pressure $B$ : for pions $p_{\text {rad }} \sim 0.02 B$. The time $\tau$ for the bag to dissolve into its radiation is

$$
\tau \simeq \frac{3 \pi \exp \left(\frac{m}{T_{\mathscr{H}}}\right) E_{0}}{g_{m} R_{0}^{2} m^{2} T_{\mathscr{H}}^{2}},
$$

$g_{m}$ is the particle degeneracy and $R_{0}$ and $E_{0}$ are the radius and total energy of the initial bag.

The fluxes written in Eqs. (6.1) (particle or energy per unit surface area) are integrated over an assumed spherical bag to give the result in Eq. (6.2). However, because of the lack of surface tension, the bag's maximum entropy corresponds to either an elongated (cylinder) or a flattened shape (disc). Thus, Eq. (6.2) should be interpreted as an upper limit.

The decoupling between the vapor concentration and $m$ and $T_{\mathscr{H}}$ occurs when $\mathscr{H}$ has completely evaporated (i.e. $E-N m-\frac{3}{2} N T_{\mathscr{H}}=0$ ) at a volume of $V_{d} \simeq 1 / n_{\mathscr{H}} E /\left(m+\frac{3}{2} T_{\mathscr{H}}\right)$. The disappearance of $\mathscr{H}$ allows the vapor concentration to decrease as $N / V=n_{\mathscr{H}} V_{d} / V$.

For $V>V_{d}$ due to energy and particle number conservation the temperature is fixed at $T_{\mathscr{H}}$. This assumes the Hagedorn spectrum extends to $m=0$. However, there may be a lower cut off at $m_{0}$ which modifies the results as follows. For energies $E-m N-\varepsilon \gg m_{0}$ and $V<V_{d}$ the above results hold. For $V \gg V_{d}$, the situation is different: $\mathscr{H}$ evaporates until its mass is $m_{0}$. If the entire mass of $\mathscr{H}$ is transformed into vapor particles as the volume increases further, then the excess particles increases the concentration and decreases the temperature. As the volume increases further, the concentration varies as $N / V=\left(n_{\mathscr{H}} V_{d}+\frac{m_{0}}{m}\right) / V$ while the temperature remains constant at $T=n_{\mathscr{H}} V_{d} /\left(n_{\mathscr{H}} V_{d}+\frac{m_{0}}{m}\right) T_{\mathscr{H}}$.

The threshold $m_{0}$ is absolute regardless of bag multiplicity. Many bags in equilibrium have a global Hagedorn threshold $m_{0}$ so particle-particle collisions are identical to heavy ion reactions.

\section{Fragmentation of $\mathscr{H}$}

One last, but not least problem is the stability of $\mathscr{H}$ against fragmentation into lighter $\mathscr{H}$ particles (shown schematically in Fig. 4). In other words, given the total mass of the initial $\mathscr{H}$ particle, we want to calculate the equilibrium mass distribution and concentrations of the system as function of volume.

At the risk of stating the obvious, if we neglect the translational phase space, the $\mathscr{H}$ particle, deprived as it is of surface terms, turns out to be totally indifferent to fragmentation. 


\section{Stability of the Hagedorn bag against fragmentation}

-If no translational or positional entropy, then the Hagedorn bag is indifferent to fragmentation.
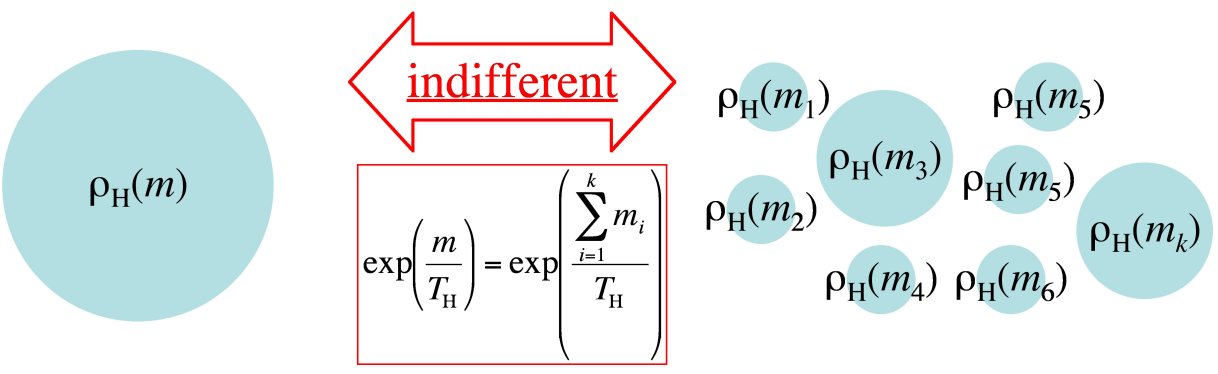

Figure 4: Schematic representation of the fragmentation o $\mathscr{H}$.

On the other hand, the translational degrees of freedom can be properly accounted for by introducing the Hagedorn weight $\exp \left(\frac{m}{T_{\mathscr{H}}}\right)$ in Eq. (3.2). Following exactly the same procedure we arrive at the equivalent of Eq. (3.5) for each value of $\mathscr{H}$ mass $m$ :

$$
\frac{N(m)}{V}=\left(\frac{m T_{\mathscr{H}}}{2 \pi}\right)^{\frac{3}{2}} .
$$

This gives the equilibrium value of particle density for $\mathscr{H}$ of mass $m$. Energy/mass conservation

$$
E=\int_{m_{\min }}^{m_{\max }} \frac{d m}{M} N(m)\left(m+\frac{3}{2} T_{\mathscr{H}}\right)=V \int_{m_{\min }}^{m_{\max }} \frac{d m}{M}\left(\frac{m T_{\mathscr{H}}}{2 \pi}\right)^{\frac{3}{2}}\left(m+\frac{3}{2} T_{\mathscr{H}}\right),
$$

determines an upper cut off $m_{\max }$ for the Hagedorn mass spectrum ( $M$ is a normalization constant).

Given $E$, the total mass/energy of the initial $\mathscr{H}$ particle, Eq. (7.2) is an implicit equation for mass vs. $E$. The physical implication of these results is interesting:

1) Since the Hagedorn is assumed to split only into other Hagedorns at infinitum, a gas of Hagedorns must be at saturation with itself, i.e. the concentrations of the various masses below $m_{\max }$ cannot change with volume, and, of course, the temperature remains fixed at $T_{\mathscr{H}}$;

2) However, as the volume increases/decreases, the upper cut-off $m_{\max }$ decreases/increases according to the conservation law expressed by (7.2).

In any case, the distribution is dominated by the largest Hagedorn mass $m_{\max }$. We can also, trivially, define the Hagedorn gas equation of state, which for $m_{\min }=0$ acquires a simple form

$$
N_{\text {tot }}=\int_{0}^{m_{\max }} \frac{d m}{M} N(m)=\frac{2 m_{\max }}{5 M} V\left(\frac{m_{\max } T_{\mathscr{H}}}{2 \pi}\right)^{\frac{3}{2}}, \quad P=\frac{N_{t o t} T_{\mathscr{H}}}{V}, \quad \frac{E}{V}=\frac{3}{2} P+\gamma P^{\frac{7}{5}}
$$

An example of the pressure is given in Fig. 5. 


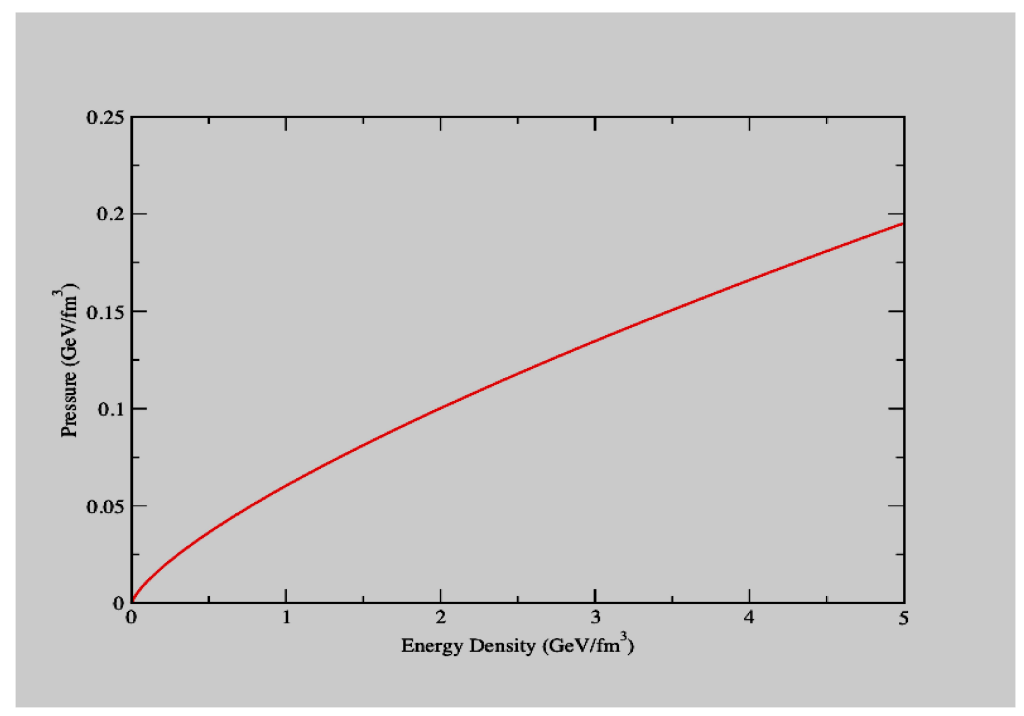

Figure 5: An example of the pressure as a function of energy density is given for the Hagedorn equation of state. See Eq. (7.3)

Here the constant $\gamma$ is defined as

$$
\gamma=\frac{5}{7 T_{\mathscr{H}}^{2}}\left[50 \pi^{3} M^{2}\right]^{1 / 5}
$$

From the equation of state (7.3) one can determine the speed of sound $c_{s}$ (see Fig. 6) as

$$
c_{s}^{2}=V \frac{d P}{d E}=1 /\left[\frac{3}{2}+\gamma P^{2 / 5}\right] .
$$

The latter vanishes in the high pressure limit or at small volumes $V \rightarrow 0$ and fixed $E$.

\section{The Bag Surface}

The bag expressions reported above contain only volume terms. Given the finite size of the bags that are interpreted as resonances, it may be of interest to consider finite size effects and their role in the description of the bags properties. The simplest generalization, assuming that the bags are leptodermous (which is supported by the short range of hadron-hadron interaction) is that of introducing a surface energy.

Thus, the pressure of a spherical bag can be written as

$$
p=\frac{\sigma}{3} T^{4}-B-a_{s}(T) V^{-\frac{1}{3}}=\frac{\sigma}{3} T^{4}-B-\frac{a_{s}(T)}{\alpha R},
$$

where $a_{s}(T)$ is the temperature dependent surface energy coefficient, $R$ is the bag radius and $\alpha \equiv$ $\left[\frac{4 \pi}{3}\right]^{\frac{1}{3}}$. Using the thermodynamic identities for the free energy $F$ and entropy $S$

$$
p=-\left(\frac{\partial F}{\partial V}\right)_{T}, \quad \text { and } \quad S=-\left(\frac{\partial F}{\partial T}\right)_{V},
$$




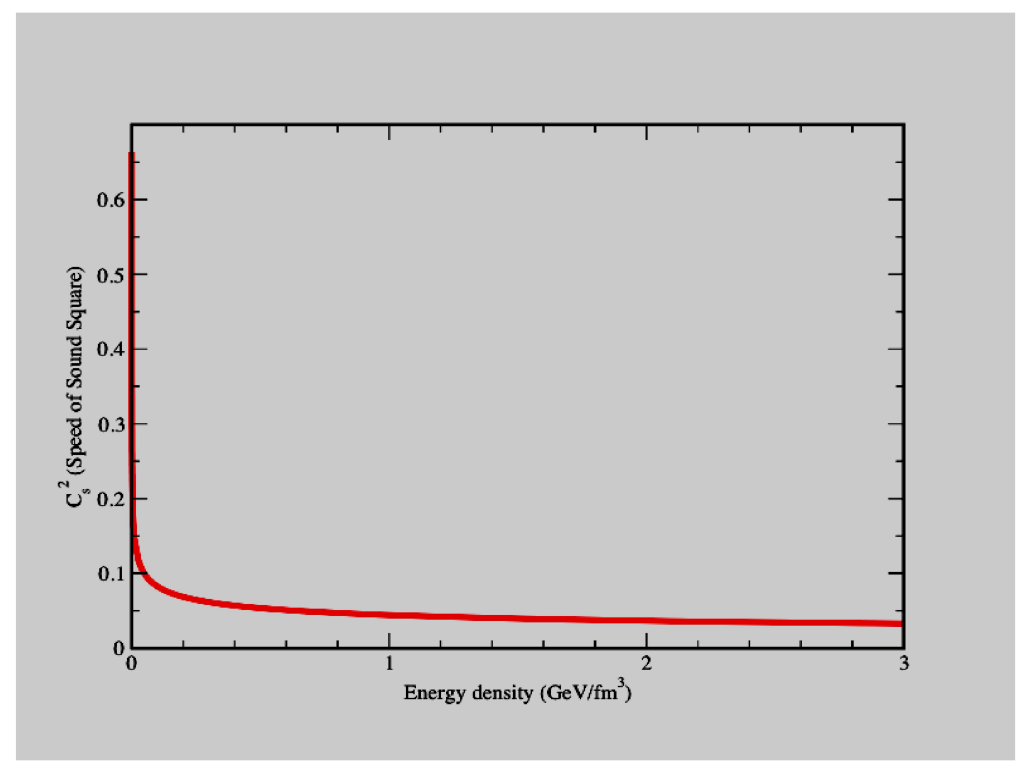

Figure 6: The square of the speed of sound as a function of energy density.

one can find all thermodynamic functions as follows

$$
\begin{aligned}
F & =-\left[\frac{\sigma}{3} T^{4}-B\right] V+\frac{3}{2} a_{s}(T) V^{\frac{2}{3}}, \\
S & =\frac{4 \sigma}{3} T^{3} V-\frac{3}{2} \frac{d a_{s}(T)}{d T} V^{\frac{2}{3}}, \\
\varepsilon V & =\left[\sigma T^{4}+B\right] V+\frac{3}{2}\left[a_{s}(T)-\frac{d a_{s}(T)}{d T}\right] V^{\frac{2}{3}} .
\end{aligned}
$$

In evaluating the expression (8.3) we fixed the integration constant (an arbitrary function of $T$ ) to zero because for the bag of zero volume the free energy should vanish.

While theoretical input on the magnitude of $a_{s}(T)>0$ is needed the consequences associated with the possible existence of this surface term are surprising. In Eq. (8.1) the surface term appears as an additional pressure to the bag pressure. Therefore, setting the total pressure to zero $p=0$, we obtain for the bag temperature

$$
T=T(R)=\left[\frac{3}{\sigma}\left(B+\frac{a_{s}(T)}{\alpha R}\right)\right]^{\frac{1}{4}} .
$$

When $R$ is large we recover the previous bag temperature and the associated physics. When $R$ becomes small, however, the bag temperature increases! The implications of his dependence are strange indeed. The first is the peculiar behavior of the bag's heat capacity. The second is the stability of the gas of bags (or lack thereof). The third is the signature of a bag's decay.

\section{Heat Capacity}

In the standard bag model the heat capacity is infinite: no matter how much energy is fed to the bag, its temperature remains constant $[10,13]$. The only effect is to make the bag larger. This is 
completely consistent with what we observe in isobaric phase transitions in ordinary matter. Here the isobaric condition is produced by the bag constant, and the phase transition is from hadronic to partonic phase.

Surface effects, however, lead to an apparently non-thermodynamic behavior: the more we feed the energy to the bag, the lower its temperature becomes. This can be stated by saying that the bag's heat capacity is negative.

\section{Stability of a Gas of Bags}

We have shown above that an ordinary bag (no surface energy) is nearly indifferent to fragmentation into smaller bags. In fact, under rather general conditions it appears that there is a mild tendency for a gas of bags to collapse into a single one. We show now that the introduction of the surface energy leads to an even stronger tendency for a gas of bags toward collapse.

Let us assume an arbitrary mass distribution in a gas of bags, and for simplicity, let us assume that the gas is confined in a fixed volume along with its decay products (say pions). The gas cannot be isothermal since the smaller bags have larger temperatures than the big ones. Thus the smaller bags evaporate first and their evaporation products are absorbed by the larger bags until only one remains. It may be argued that isothermicity can be achieved by having all the bags to be of the same size. But this situation is clearly unstable. Any small perturbation in size will lead to a catastrophic collapse of all bags into a single one.

\section{Decay of a Bag}

A bag, unless constrained by conserved quantities, must decay. As it decays, the instantaneous spectrum of the decay products will indicated the bag's instantaneous temperature. Without surface effects the bag temperature is constant and the overall spectrum and the instantaneous spectrum will be the same.

With the surface effects, as the bag decays and becomes smaller, its temperature increases. Therefore the overall spectrum integrated over the overall decay must differ from the instantaneous spectrum associated with each temperature. The shape deviation of the overall spectrum from that of an instantaneous spectrum at fixed temperature may be an interesting observable to characterize both the effect and the magnitude of the surface energy coefficient. It is amusing to notice the similarities with the decay of a black hole through Hawking radiation.

\section{Conclusions}

An $\mathscr{H}$ system is a perfect thermostat at fixed temperature $T_{\mathscr{H}}$ and a perfect particle reservoir. Particles in equilibrium with or emitted by $\mathscr{H}$ are in physical and chemical equilibrium with themselves and with $\mathscr{H}$. They constitute a saturated vapor. This defines a first order phase transition and a phase coexistence completely controlled by the bag pressure. The hadronic side of $\mathscr{H}$ radiates particles in preexisting physical and chemical equilibrium just as a black body radiates photons in physical and "chemical" equilibrium. An $\mathscr{H}$ system is nearly indifferent to fragmentation into smaller $\mathscr{H}$ systems. This near indifference to fragmentation makes this work relevant 
to elementary particle and heavy ion collisions. The equation of state for a gas of Hagedorns has been derived. A lower cut off in the mass spectrum does not alter our results. The introduction of surface energy makes the smaller bags hotter than the larger ones. This completely destabilizes a gas of bags, which would collapse into a single bag.

\section{Acknowledgments}

We thank V. Koch for his encouragement and attention and J. I. Kapusta for pointing out ref. [11]. This work was supported by the Director, Office of the Energy Research, Office of High Energy and Nuclear Physics, Division of Nuclear Physics, of the US Department of Energy under Contract No. DE-AC03-76SF00098.

\section{References}

[1] R. Hagedorn, Suppl. Nuovo Cimento 3, 147 (1965).

[2] R. Hagedorn and J. Ranft, Suppl. Nuovo Cimento 6, 169 (1968).

[3] D. B. Blaschke and K. A. Bugaev, Prog. Part. Nucl. Phys. 53, 197 (2004).

[4] A. Chodos et. al., Phys. Rev. D 9, 3471 (1974).

[5] J. I. Kapusta, Phys. Rev. D 10, 2444 (1981).

[6] J. I. Kapusta, Nucl. Phys. B 196, 1 (1982).

[7] T. Alexopoulos et al, Phys. Lett. B 43, 528 (2002).

[8] M. Kliemant, B. Lungwitz and M. Gazdzicki, Phys. Rev. C 69, 044903 (2004).

[9] F. Becattini and L. Ferroni, Acta Phys. Polon. B 35, 2007 (2004).

[10] L. G. Moretto, K. A. Bugaev, J. B. Elliott, and L. Phair, Europhys. Lett. 76, 402 (2006) .

[11] R. D. Carlitz, Phys. Rev. D 5, 3231 (1972).

[12] F. Karsch, Nucl. Phys. Proc. Suppl 83, 14 (2000).

[13] K. A. Bugaev et al., arXiv:hep-ph/0504011.

[14] W. Broniowski, W. Florkowski and L. Y. Glozman, arXiv:hep-ph/0407290.

[15] S. C. Frautschi, Phys. Rev. D 3, (1971) 2821; C. J. Hamer and S. C. Frautschi, Phys. Rev. D 4, (1971) 2125.

[16] R. K. Pathria, Statistical Mechanics, Pergamon Press, Oxford 1972.

[17] K. A. Bugaev, Nucl. Phys. A 606, 559 (1996).

[18] K. A. Bugaev, M. I. Gorenstein and W. Greiner, J. Phys. G 25, 2147 (1999). 